\title{
Scutellaria baicalensis Inhibits Coxsackievirus B3-Induced Myocarditis Via AKT and p38 Pathways
}

\author{
Qiang $\mathrm{Fu}^{1 \dagger}$, $\mathrm{Lu} \mathrm{Gao}^{2 \dagger}$, Xiao $\mathrm{Fu}^{3}$, Qinghua Meng ${ }^{4,5,6}$, and Zhihong $\mathrm{Lu}^{7 *}$ \\ ${ }^{1}$ Department of Cardiovascular Surgery, General Hospital of Tianjin Medical University, Tianjin 300070, P.R. China \\ ${ }^{2}$ Department of Cardiovascular Medicine, Tianjin Nankai Hospital, Tianjin, P.R. China \\ ${ }^{3}$ Department of Biochemistry and Molecular Biology, School of Basic Medical Sciences, Tianjin Medical University, Tianjin 300070, P.R. China \\ ${ }^{4}$ College of Physical Education and Educational Sciences, Tianjin University of Sport, Tianjin 301617, P.R. China \\ ${ }^{5}$ Tianjin Key Laboratory of Sports Physiology and Sports Medicine, Tianjin University of Sport, Tianjin 301617, P.R. China \\ ${ }^{6}$ Department of Physical Education, Tsinghua University, Beijing 100084, P.R. China \\ ${ }^{7}$ Department of Anatomy and Histology and Embryology, School of Basic Medical Sciences, Tianjin Medical University, Tianjin 300070, P.R. China
}

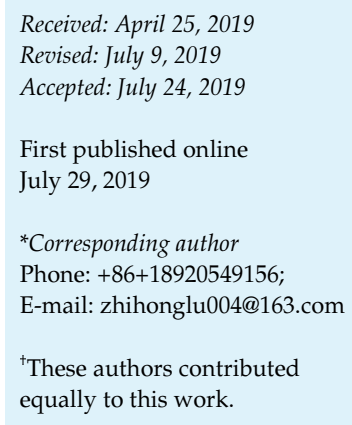

Scutellaria baicalensis Georgi has been widely used in China for treatment of various diseases. This study investigated the effect of Scutellaria baicalensis Georgi extracts (SBE) against Coxsackievirus B3 (CVB3)-induced myocarditis in vitro and in vivo. In vitro, Hela cells and primary myocardial cells were infected with CVB3 and treated with SBE $(50-800 \mu \mathrm{g} / \mathrm{ml})$ and ribavirin $(200 \mu \mathrm{M})$ for $48 \mathrm{~h}$ and then determined by CCK 8 assay. Real-time PCR and western blotting assays were performed. In vivo, a myocarditis model was induced in male BALB/c mice by injecting CVB3 suspension intraperitoneally for three times, followed by treatment with SBE (400 and $200 \mathrm{mg} / \mathrm{kg}$ ) and ribavirin $(100 \mathrm{mg} / \mathrm{kg})$ for 28 days. SBE ameliorated the cytotoxicity of CVB3 in Hela cells, especially at $400 \mu \mathrm{g} / \mathrm{ml}(39.93 \% v s 65.67 \%, p<0.05)$ without influencing cell growth and also significantly reduced CVB3 replication in primary myocardial cells. The levels of AKT, ERK, and p38 were increased after CVB3 infection. SBE could downregulate the expressions of AKT and p38. In vivo, the mortality rate from CVB3 reached to $66.67 \%$, while $10.00 \%$ and $23.33 \%$ of this came after 400 and $200 \mathrm{mg} / \mathrm{kg} \mathrm{SBE}$ treatment, respectively $(p<0.05)$. The CVB3 replication was obviously reduced after SBE administration from day 5. Similarly, the levels of AKT, ERK, and p38 mRNAs and proteins were increased, and SBE suppressed the expression of AKT and p38. Our study indicates that SBE is a promising potent antiviral agent against CVB3-induced myocarditis by inhibition of virus replication via depressing AKT and p38 expressions.

Keywords: Scutellaria baicalensis, Coxsackievirus B3, myocarditis, AKT, p38

\section{Introduction}

Myocarditis represents one of the most challenging clinical problems in cardiology, and can cause mild dyspnea, acute heart failure and sudden death [1]. Viral myocarditis is a common form of myocarditis and is characterized by cardiac inflammation. It is a triphasic disease involving an initial viral infection, followed by an autoimmune response, and results in the remodeling of the cardiac structure and function [2]. Coxsackievirus B3
(CVB3) is a relatively common enterovirus that typically leads to mild upper respiratory and gastrointestinal illness, and it is the most frequent etiological agent to induce myocarditis, which is life-threatening due to the risk of heart failure, with a poor prognosis in cases of acute and chronic myocarditis [3]. CVB3 proliferates rapidly within human cardiomyocytes, activates the antimicrobial hostdefense pathways, and thus results in the production of heart-reactive autoantibodies. In other words, CVB3 infection induced damage to the heart and the host immune 
response is a main process in the pathogenesis of CVB3related myocarditis $[3,4]$. The pathogenesis of CVB3induced myocarditis may relate to the activation of caspase-3, AMPK/MEK/ extracellular signal-regulated kinase (ERK), protein kinase B/AKT (PKB/AKT), endoplasmic reticulum (ER) stress, p38 and phosphatidylinositol 3kinase $(\mathrm{PI} 3 \mathrm{~K}) / \mathrm{AKT} / \mathrm{mammalian}$ target of rapamycin (mTOR) pathways [5-7]. These pathways can involve autophagy or apoptosis in host cells after CVB3 infection.

Scutellaria baicalensis Georgi, a traditional Chinese medicinal herb, has been widely used in China for thousands of years and shows various activities in treating diarrhea, dysentery, hypertension, cancer, influenza, bacterial pneumonia, inflammation and respiratory infections [8-10]. In the roots of Scutellaria baicalensis, flavones such as baicalin and wogonoside and their aglycones baicalein and wogonin are the major bioactive compounds, having been reported to demonstrate various pharmacological functions, including anticancer, hepatoprotection, antibacterial and antiviral, antioxidant, anticonvulsant and neuroprotective effects. Thus far, there are only a few studies that have investigated the effects of the active components of Scutellaria baicalensis such as oroxylin A [11]. The effect of Scutellaria baicalensis on CVB3-induced myocarditis is not reported. Therefore, our study was designed to determine whether it showed the anti-CVB3 virus activity in vitro and in vivo. Some reports found that Scutellaria baicalensis can influence the $\mathrm{PI} 3 \mathrm{~K} / \mathrm{AKT} / \mathrm{NF}-\kappa \mathrm{B}, \mathrm{MAPK} / \mathrm{ERK}$, and p38 signaling pathways $[9,10]$.

There are several host cells for CVB3 infection in vitro experiments such as HeLa, Vero cells, and neonatal rat cardiac fibroblasts. In the current study, we selected rat primary myocardial cells as the host cells. It has been reported that viral myocarditis mice models have been established through intraperitoneally injecting mice with CVB3. We also used the same myocarditis model to determine the effect of Scutellaria baicalensis on CVB3-induced damage in mice. Additionally, Scutellaria baicalensis extract (SBE) contains the bioactive components of Scutellaria baicalensis. Our study showed that SBE had significant antiviral activities against CVB3 infection in vitro and in vivo, which was associated with a reduced level of CVB3induced cytotoxicity. The expression of AKT, ERK, and p38 mRNAs and proteins were increased after CVB3 infection in host cells and mice, but SBE treatment significantly suppressed the elevation of AKT and p38 genes. The results indicated that $\mathrm{SBE}$ has the potential to be an antiviral agent against CVB3 infection.

\section{Materials and Methods}

\section{Animals}

Male BALB/c mice (6-weeks-old) were obtained from the Sbef Biotechnology Co., Ltd. (China). The animals were housed in polycarbonate cages with white wood chips for bedding, and given free access to food and drinking water, under controlled temperature $\left(23 \pm 2^{\circ} \mathrm{C}\right)$, humidity $(50-60 \%)$ and photoperiod $(12 \mathrm{~h}$ light and $12 \mathrm{~h}$ dark). All animal experiments were performed according to the Guide for the Care and Use of Medical Laboratory Animals (Ministry of Health, People's Republic of China) and with the ethical approval of the Animal Care and Use Committee of Chinese Academy of Medical Sciences Institute of Radiation Medicine.

\section{Virus Strain, Cells and Plant Extract}

Nancy strain coxsackievirus group B type 3 (CVB3) was purchased from the Wuhan Institute of Virology, Chinese Academy of Sciences (China). CVB3 was propagated in HeLa cells and it was maintained and stored at $-80^{\circ} \mathrm{C}$. The amplified CVB3 titer was determined by the plaque-forming assay in HeLa cells and a $50 \%$ tissue culture infectious dose $\left(\mathrm{TCID}_{50}\right)$ was calculated. HeLa cells were grown and maintained in RPMI-1640 supplemented with $10 \%$ fetal bovine serum (FBS, Hyclone). Primary rat myocardial cells were obtained from neonatal rat hearts and cultured with $\alpha$-MEM containing $10 \%$ (vol/vol) FBS. SBE was obtained from Xi'an Ming Ze Biological Technology Co., Ltd. (China), which contains $85 \%$ of baicalin.

\section{Anti-CVB3 in Vitro Assays}

Hela cells were seeded in a 12-well culture plate with the density of $2.0 \times 10^{5}$ cells/well. When the cells reached a confluence of $80 \%$, the cells were infected with CVB3 $\left(100 \times \mathrm{TCID}_{50}\right)$ [12]. After infection, various concentrations of SBE (final concentrations were $50,100,200,400$, and $800 \mu \mathrm{g} / \mathrm{ml}$ ) and ribavirin (final concentrations were $200 \mu \mathrm{M}$ ) were added to the medium. Hela cells and CVB3 infection cells were used as the normal group and model group, respectively, and treated with physiological saline. The cells were incubated at $37^{\circ} \mathrm{C}$ with $5 \% \mathrm{CO}_{2}$ for $48 \mathrm{~h}$. The cell viability on every plate was detected using the Cell Counting Kit 8 (CCK8) (Dalian Meilun Biotechnology Co., LTD., China) and the OD was determined at $450 \mathrm{~nm}$ using an enzyme-linked immunosorbent assay reader (Bio-Rad, USA). The proliferation rate was calculated using the following equation:

Cell viability rate $=\left[\left(\mathrm{OD}_{\mathrm{T}}-\mathrm{OD}_{\mathrm{B}}\right) /\left(\mathrm{OD}_{\mathrm{C}}-\mathrm{OD}_{\mathrm{B}}\right)\right] \times 100 \%$,

where $\mathrm{OD}_{\mathrm{T}}, \mathrm{OD}_{\mathrm{B}}$, and $\mathrm{OD}_{\mathrm{C}}$ denote the absorbencies of the tested compounds, blank, and solvent control, respectively. The cell viability of the untreated cells was $100 \%$. Three independent replicates were performed in this experiment.

Primary myocardial cells were infected by CVB3 for 2 days and then various concentrations of SBE (final concentrations were 50, $100,200,400$, and $800 \mu \mathrm{g} / \mathrm{ml}$ ) were added. The virus supernatants were collected to calculated $-\log _{10} \mathrm{TCID}_{50}$. 


\section{Murine CVB3 Myocarditis Animal Model}

According to the reported methods [12], male BALB/c mice were inoculated intraperitoneally with $0.1 \mathrm{ml} 1 \times 10^{3} \mathrm{TCID}_{50} \mathrm{CVB} 3$ suspension for three consecutive times (once a day). Mouse survival was recorded upon 28 days post-infection at termination of the experiment. The viral myocarditis mice were divided into four groups of 30 mice per group: 1) Normal control group (Normal), $0.9 \%$ saline solution, $10 \mathrm{ml} / \mathrm{kg} / \mathrm{d}$ once daily via intra-gastric administration after injection $0.1 \mathrm{ml} 0.9 \%$ saline solution; 2) viral myocarditis group (Model), $0.9 \%$ saline solution, $10 \mathrm{ml} / \mathrm{kg} / \mathrm{d}$ once daily via intra-gastric administration after injection CVB3 suspension; 3) ribavirin group (Ribavirin), $100 \mathrm{mg} / \mathrm{kg} / \mathrm{d}$ once daily via intra-gastric administration after injection CVB3 suspension; 4) high-dose SBE group (SBE-H) consisting of $400 \mathrm{mg} / \mathrm{kg} / \mathrm{d}$ once daily via intra-gastric administration after injection CVB3 suspension; 5) low-dose SBE group (SBE-L), $200 \mathrm{mg} / \mathrm{kg} / \mathrm{d}$ once daily via an intra-gastric administration after injection CVB3 suspension. Treatment began $24 \mathrm{~h}$ after the final injection of CVB3 and was performed once per day for 28 days. On day 5 , and 12 after the injection of CVB3, three mice per group were sacrificed and heart tissue was weighed. TCID 50 of CVB3 were determined on day 5 . The ratio of heart weight to body weight (heart index) was calculated on day 5, 12, and 28. On d 28, heart tissue was collected for histopathological analysis. Heart inflammation level was observed by hematoxylin and eosin (H\&E). The remaining nine mice per group continued to be fed and monitored for weight loss and mortality until the end of the experiment.

\section{Quantitative Real-Time PCR (qRT-PCR)}

To evaluate the effect of treatments on mRNA levels of AKT, ERK, and p38 in primary myocardial cells, the cells were collected after $200 \mu \mathrm{M}$ ribavirin, $100 \mu \mathrm{g} / \mathrm{ml}$ or $400 \mu \mathrm{g} / \mathrm{ml} \mathrm{SBE}$ treatment (SBE-L, SBE-H) for $48 \mathrm{~h}$. In myocarditis mouse model, the myocardial tissues of each group after treatment with ribavirin and SBE for $28 \mathrm{~d}$ were prepared. Total mRNAs from myocardial cells and heart tissues were isolated using $1 \mathrm{ml}$ TRIzol reagent according to the protocol, followed by reverse transcription of total RNA to cDNA. A Prime Script and RT Reagents Kit (perfect real time) was purchased from ABI-Invitrogen (USA). cDNA subsequently underwent quantitative real-time polymerase chain reaction (PCR). All primer pairs produced a single PCR product as determined by the dissociation curve and gel analysis. Three samples were measured in each experimental group in quadruplicate, with a minimum of two independent experiments. Reactions of the PCR were carried out using the forward primer and reverse primer as shown in Table 1. An individual reaction was performed using the ABI 7900 Real-Time PCR System (Thermo Fisher Scientific, USA) with reverse transcription at $42^{\circ} \mathrm{C}$ for $5 \mathrm{~min}$, followed by enzymatic activation at $95^{\circ} \mathrm{C}$ for $2 \mathrm{~min}$, denaturation for $20 \mathrm{sec}$ at $94^{\circ} \mathrm{C}$, and elongation for up to $20 \mathrm{sec}$ at $60^{\circ} \mathrm{C}$. The relative amount of target mRNA normalized to actin was calculated. The expression rates of AKT, ERK, and p38 were counted using the formula: $2(-\Delta \Delta \mathrm{CT})$, with $\Delta \mathrm{CT}=\mathrm{PCR}$ score of AKT, ERK, or p38 score actin. $\Delta \Delta \mathrm{CT}=\Delta \mathrm{CT}$ ofdruggroup $-\Delta \mathrm{CT}$ of normalgroup.

\section{Western Blot Analysis}

Primary myocardial cells treated with $200 \mu \mathrm{M}$ ribavirin, $100 \mu \mathrm{g} / \mathrm{ml}$ or $400 \mu \mathrm{g} / \mathrm{ml} \mathrm{SBE}$ treatment (SBE-L, SBE-H) for $48 \mathrm{~h}$ were collected. The cardiac muscle tissues of each group were collected in myocarditis mouse model at $28 \mathrm{~d}$. The total cells and tissue protein concentrations were detected by a BSA protein assay, separated by SDS-PAGE gel and transferred onto a PVDF membrane. Next, they were probed with specific primary antibodies, followed by the appropriate HRP conjugated sheep anti-mouse or anti-rabbit IgG antibodies (MDL Biotech, China). The antibodies used in this study were anti-AKT, anti-p-AKT, anti-ERK, anti-p-ERK, anti-p38, anti-p-p38, and $\beta$-actin served as the protein loading control. All the antibodies were purchased from MDL (China). Image J software was used to calculate the densitometric analysis of protein bands.

\section{Statistics}

The data are expressed as the mean \pm standard deviation (SD). Student's $t$-test was used to compare differences between two groups and one-way ANOVA analysis with post hoc tests was used to compare differences between group comparisons. The survival rates of CVB3-infected mice were compared and analyzed with a Kaplan-Meier plot. A value of $p<0.05$ was considered to indicate a statistically significant difference.

\section{Results}

\section{SBE Attenuated CVB3-Induced Cytotoxicity}

We evaluated the toxicity of SBE to Hela cells and found that SBE even at $800 \mu \mathrm{g} / \mathrm{ml}$ did not affect the cell growth

Table 1. The forward and reverse primers of the target gene.

\begin{tabular}{llc}
\hline Symbol & Forward primer & Reverse primer \\
\hline$\beta$-actin & GCCCTGAGGCTCTCTTCCA & GCGGATGTCGACGTCACA \\
CAR & CTCTTCTCCCCTGGTTTCTGTA & CGGCGTCATAGCAGACAGTT \\
NF- $\mathrm{B}$ & GAGGTCTCTGGGGGTACCAT & AAGGCTGCCTGGATCACTTC \\
AKT & CCGCCTGATCAAGTTCTCCT & TTCAGATGATCCATGCGGGG \\
ERK & GCTGAAGCGCCATTCAAGTT & ACTTACACCATCTCTCCCTTGC \\
p38 & GCATCATGGCTGAGCTGTTG & GAGATAAGCAGGGGGTGTCC \\
\hline
\end{tabular}


with the cell viability over $90 \%(p>0.05)$ (Fig. 1A). CVB3 infection decreased the Hela cell viability. To investigate whether SBE could ameliorate CVB3-induced cytotoxicity, Hela cells infected with CVB3 were determined by CCK8 assay. Ribavirin was used as the positive control group. CVB3 infection significantly induced about $60.07 \%$ cell death in Hela cells at $48 \mathrm{~h}$ after virus infection compared with normal group $(p<0.05)$, whereas ribavirin and SBE treatment $(50-800 \mu \mathrm{g} / \mathrm{ml})$ attenuated the cell viability of CVB3-induced cytotoxicity in a dose-dependent manner, especially at 400 and $800 \mu \mathrm{g} / \mathrm{ml}$ with $65.67 \%$ and $61.67 \%$ cell survival rate $(p<0.05)$ (Fig. 1A). This result suggested that SBE showed antiviral effects. Then we determined the virus infection in the primary myocardial cells by detecting the TCID $_{50}$. As shown in Fig. 1B, SBE (400 and $800 \mu \mathrm{g} / \mathrm{ml}$ ) obviously decreased the virus titer $(p<0.05)$, which indicated
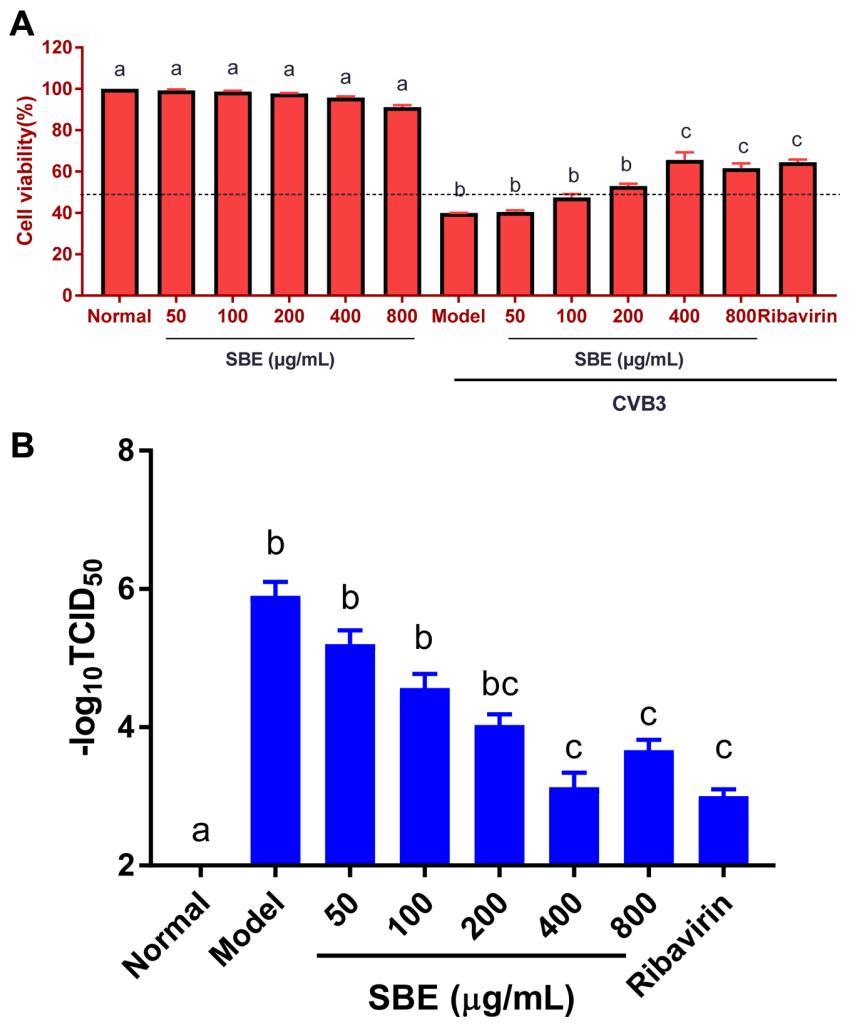

Fig. 1. The antiviral effect of SBE.

The cell viability was tested by CCK8 assay in Hela cells after CVB3 infection (A). Cytotoxicity assay of SBE on Hela cells was used to determine its toxicity. The viability of cells infected by CVB3 was significantly reduced (Model group). SBE treatment improved primary myocardial cell survival after CVB3 infection. Normal: without virus; Model: virus only. The $50 \%$ tissue culture infective dose $\left(\mathrm{TCID}_{50}\right)$ value of CVB3 in primary myocardial cells (B). Different letters represent significant differences between two groups $(p<0.05)$. that SBE could inhibit virus replication in primary myocardial cells infected by CVB3.

Effects of SBE on AKT, ERK, and p38 mRNAs and Protein Levels in Myocardial Cells

From the above results, we found the effective dose of SBE on CVB3 infection in myocardial cells is $400 \mu \mathrm{g} / \mathrm{ml}$.
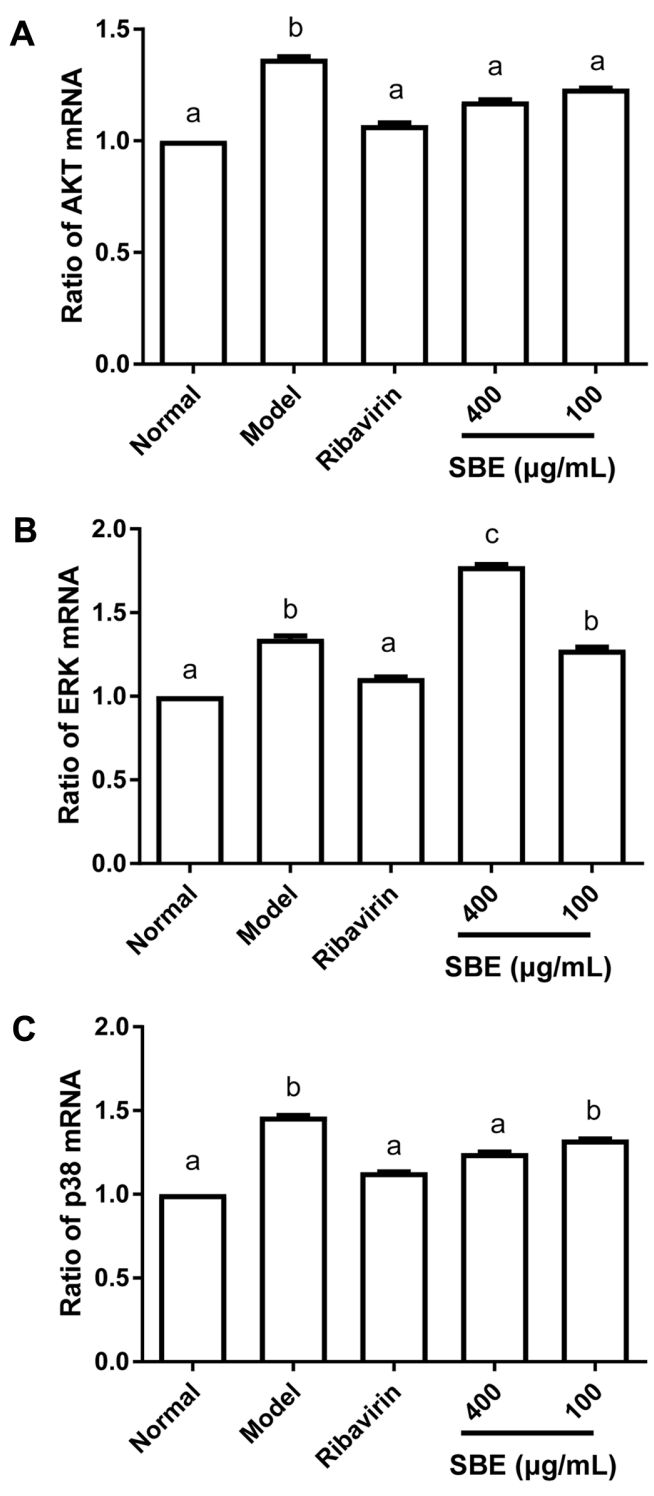

Fig. 2. Effects of SBE on mRNA levels of AKT, ERK, and p38 in primary myocardial cells after CVB3 infection.

The data shown are the RT-PCR results of relative mRNA levels of (A) AKT, (B) ERK, and (C) p38 to normal cells (without virus infection) treated with ribavirin $(200 \mu \mathrm{M})$ and SBE $(400$ and $100 \mu \mathrm{g} / \mathrm{ml})$ for $48 \mathrm{~h}$, respectively. Normal: without virus; Model: virus only. Different letters represent significant differences between two groups $(p<0.05)$. 

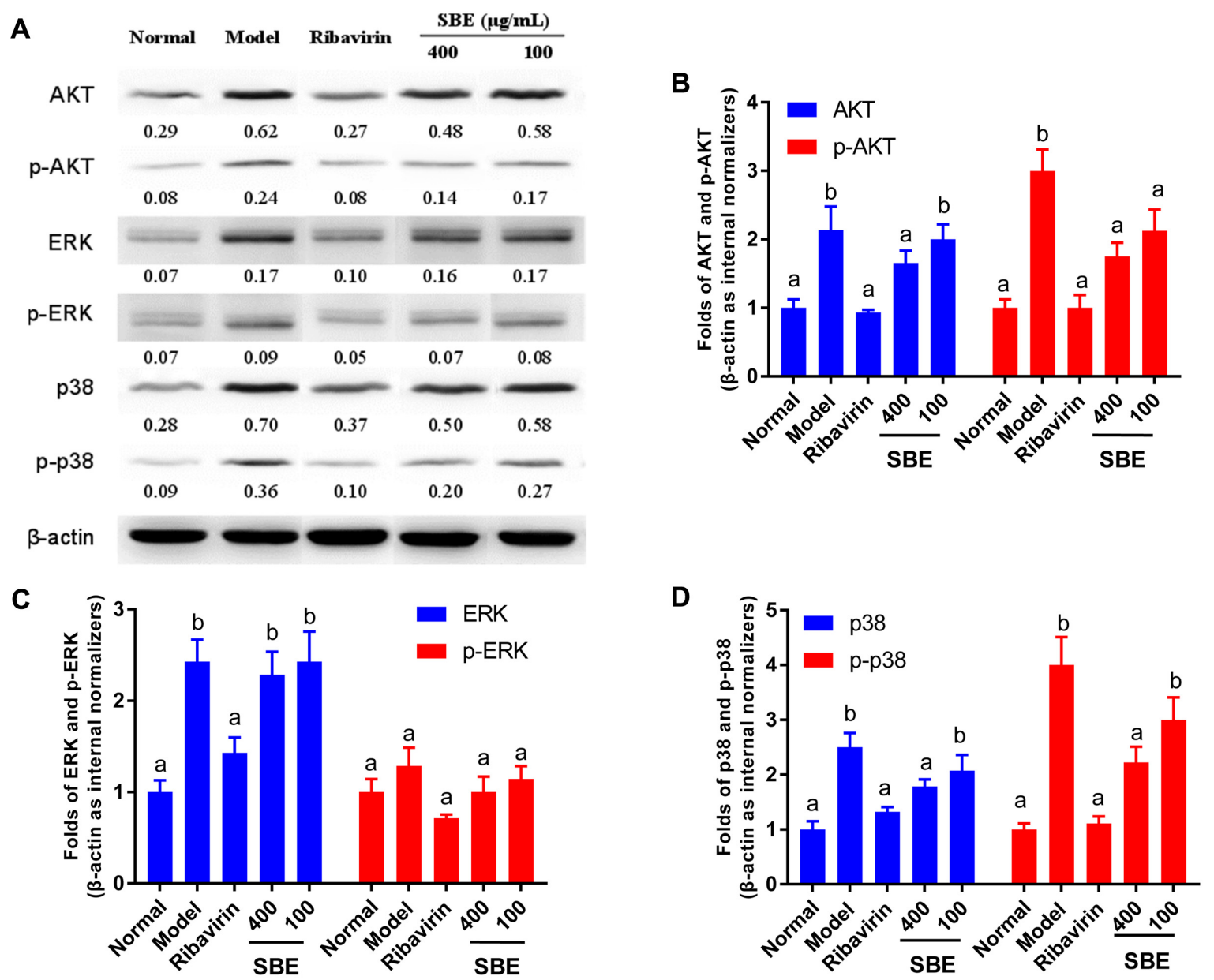

Fig. 3. SBE affected the CVB3-induced activation of AKT, ERK and p38.

(A) Representative picture of total and phosphorylated AKT, ERK and p38 protein expression. Quantitative data of AKT (B), ERK (C), and p38 (D) protein expressions. Data are expressed as fold over control ( $n=3$ per condition). Primary myocardial cells infected with CVB3 were treated with ribavirin $(200 \mu \mathrm{M})$ and SBE $(400,100 \mu \mathrm{g} / \mathrm{ml})$ for $48 \mathrm{~h}$. The levels of AKT, p-AKT, ERK, p-ERK, p38, and p-p38 proteins in cells were analyzed by western blot. Normal: without virus; Model: virus only. $\beta$-actin was used as a loading control. Different letters represent significant differences between two groups $(p<0.05)$.

Therefore, we selected 400 and $100 \mu \mathrm{g} / \mathrm{ml}$ of SBE (SBE-H, SBE-L) in the following experiments. To evaluate the mechanism of the inhibitory effect of SBE against CVB3 infection, we analyzed the mRNA and protein levels of MAPK signal pathways (Figs. 2 and 3). After $48 \mathrm{~h}$ of treatment, the expression of AKT and p38 mRNA levels was significantly enhanced in myocardial cells treated with CVB3 infection $(p<0.05)$, whereas the expressions of AKT and $\mathrm{p} 38$ genes were downregulated after ribavirin and SBE treatment $(p<0.05)$ (Figs. 2A and 2C). ERK mRNA was also improved when CVB3 infection in myocardial cells $(p<$ $0.05)$ was reversed by ribavirin. However, SBE at $400 \mu \mathrm{g} /$ $\mathrm{ml}$ further stimulated ERK expression based on CVB3 infection cells $(p<0.05)$, while SBE at $100 \mu \mathrm{g} / \mathrm{ml}$ did not influence the level of ERK mRNA ( $p>0.05$ ) (Fig. 2B).

We analyzed the proteins of AKT, ERK, and p38 upon CVB3 infection by western blot assay (Fig. 3). CVB3 infection led to increasing phosphorylation levels of AKT and p38 in myocardial cells (Figs. 3B and 3D) but failed to activate the p-ERK (Fig. 3C). However, the activation of AKT and p38 induced by CVB3 infection was significantly suppressed after ribavirin and SBE treatments compared to model group $(p<0.05)$.

\section{SBE Ameliorated the CVB3-Induced Myocarditis in Mice Model}

As shown in Fig. 4A, there is no death in the normal group. Twenty out of 30 mice died in the model group 

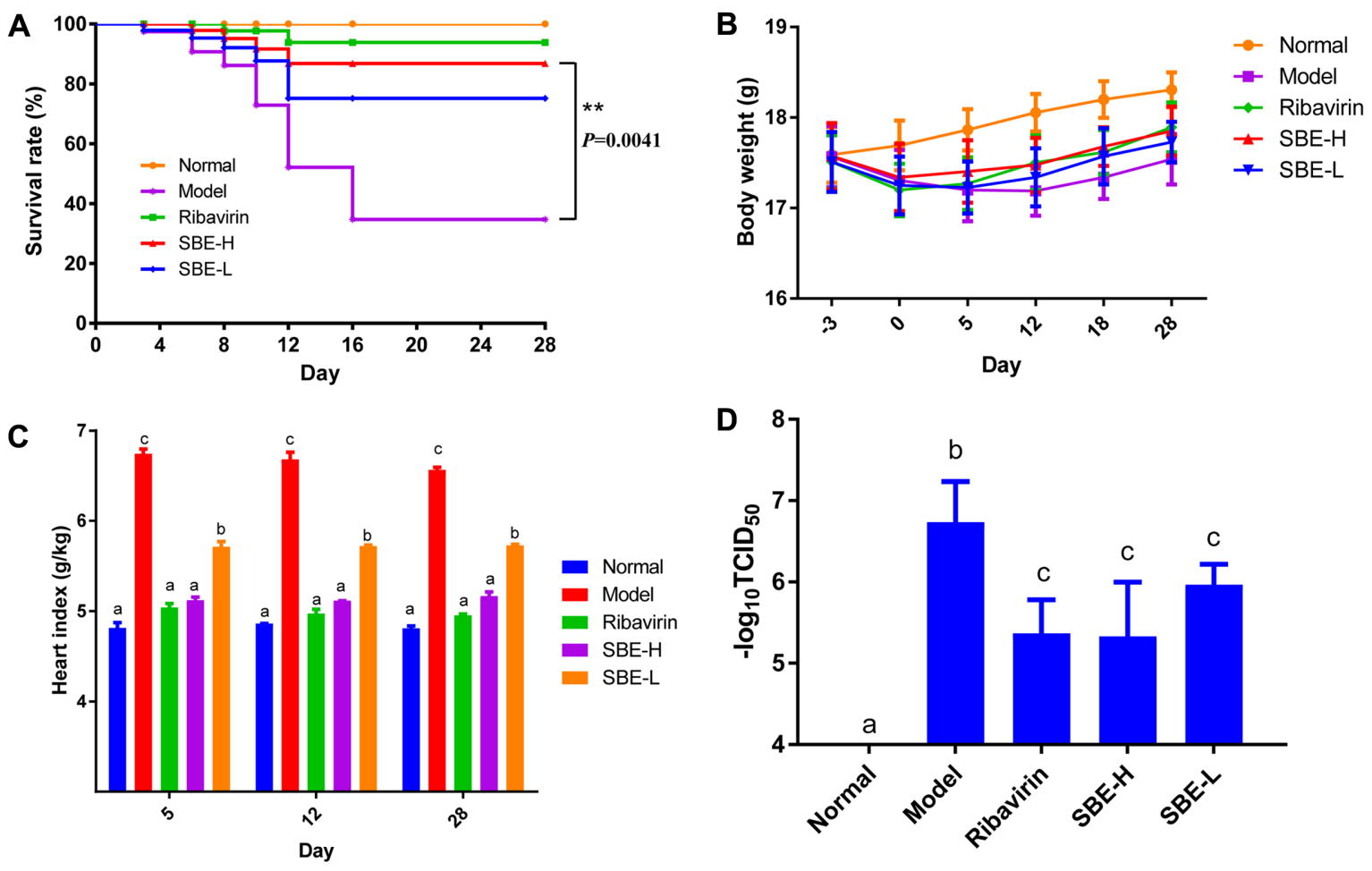

Fig. 4. (A) SBE prevents death in mice with CVB3-induced myocarditis. On day 28, animal survival rate was significantly improved in the SBE group compared with the model group (90.0\% vs. 33.33\%). (B) The body weight of different groups in the experiment. (C) The heart index of the experimental mice. (D) The 50\% tissue culture infective dose $\left(\mathrm{TCID}_{50}\right)$ value of $\mathrm{CVB}_{3}$ in the heart tissue on day 5. Different letters represent significant differences between two groups $(p<0.05)$.

(mortality rate: $66.67 \%$ ); two mice out of 30 mice died in the ribavirin-treated group (mortality rate: $6.67 \%$ ); three mice out of 30 mice died in the $400 \mathrm{mg} / \mathrm{kg}$ SBE-treated group (mortality rate: $10.00 \%$ ); seven mice out of 30 mice died in the $200 \mathrm{mg} / \mathrm{kg}$ SBE-treated group (mortality rate: $23.33 \%$ ). In the experiment, the weight of model mice after CVB3 infection was lower than the normal group, whereas SBE treatment slightly improved the index (Fig. 4B). On day 5, 12 , and 28 post infection, the severity of myocarditis was assessed using the heart index. The heart index of the model group was higher than that of normal group, while SBE groups inhibited the increasing heart index of mice induced by CVB infection (Fig. 4C). Three mice per group were sacrificed on day 5 to detect the $\mathrm{TCID}_{50}$ of CVB3 in the heart tissues. As shown in Fig. 4D, all of the SBE treatment groups and the ribavirin group showed significantly reduced the $\mathrm{TCID}_{50}$. A histopathological evaluation was performed to detect the presence and severity of myocarditis in the heart tissues of the different groups. Infected hearts revealed typical lesions of mononuclear cellular infiltration and necrosis. In contrast, no inflammatory reaction was observed in the normal mice. On day 5 and 10, the tissue damage in the SBE groups was significantly reduced compared with the model group (Fig. 5).

\section{The Regulation of AKT, ERK, and p38 in CVB3 Myocarditis Mice Model}

To identify whether the activity of SBE on the improvement of CVB3-induced myocarditis is associated with MAPK signal pathways, we evaluated the expression of AKT, ERK, and p38 mRNAs and proteins in myocardial tissues (Figs. 6 and 7). Consistent with the in vitro test, the model group obviously had elevated levels of AKT, ERK, and p38 mRNAs compared to normal group $(p<0.05)$. Ribavirin and SBE (400 mg/kg) suppressed the expressions of AKT and p38 mRNA ( $p<0.05$, Figs. 6A and 6C) when compared with the model group, but not ERK mRNA (Fig. 6B).

Western blot results showed that the total and phosphorylation levels of AKT, ERK and p38 were significantly increased in cardiac muscle tissues in the model group $(p<0.05)$ (Fig. 7). Similarly, the positive drug 


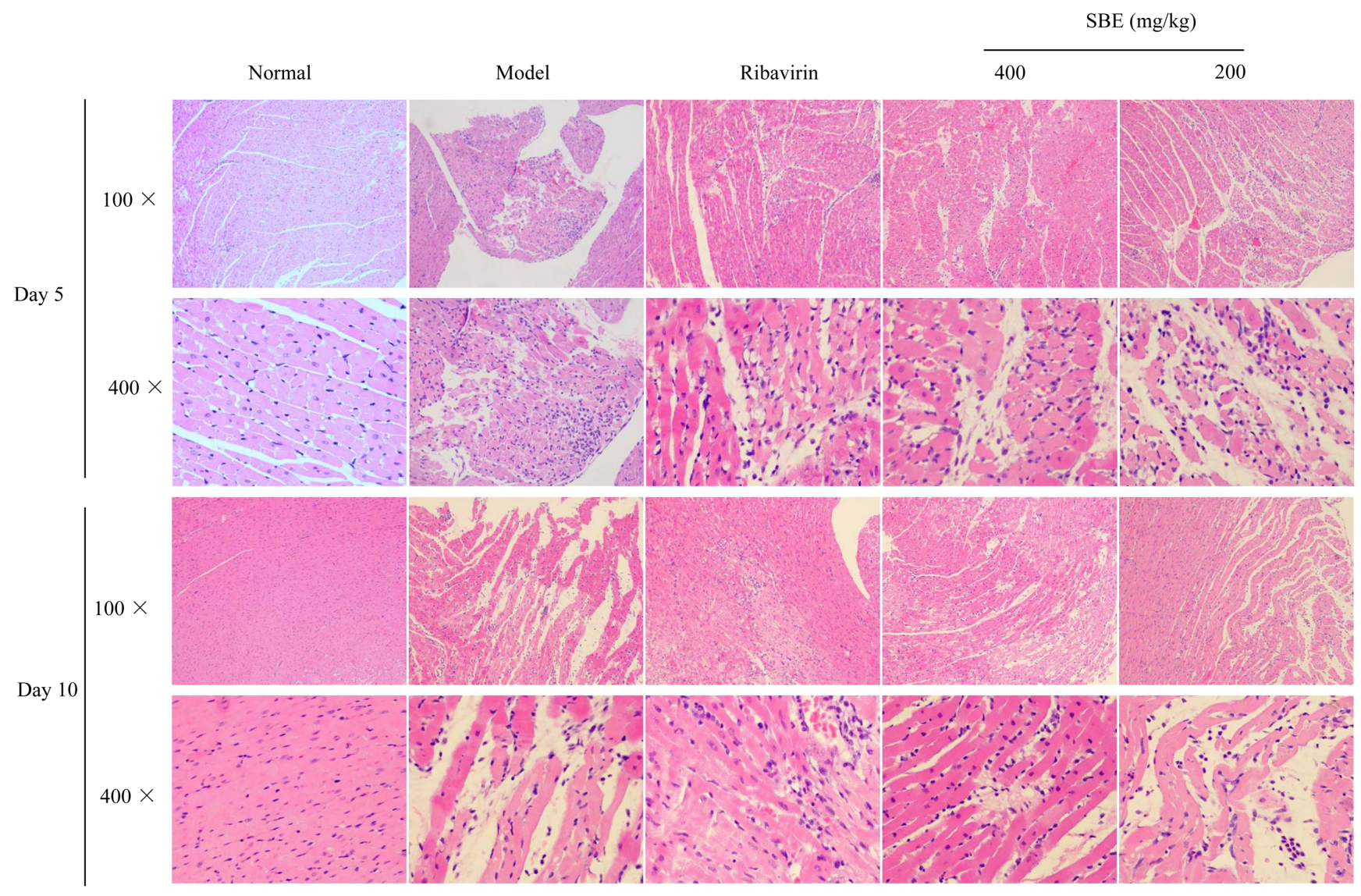

Fig. 5. SBE demonstrated protective effects on the pathological changes induced by CVB3 infection.

Representative images of HE staining on day 5 and 10.

ribavirin and SBE treatment $(400 \mathrm{mg} / \mathrm{kg})$ down-regulated the expressions of AKT and $\mathrm{p} 38$ in cardiac muscle tissues $(p$ $<0.05$, Figs. 7B and 7D). The levels of ERK were slightly suppressed by the SBE treatment ( $p>0.05$, Fig. 7C). The results suggested that SBE caused the inhibition of the CVB3-induced myocarditis and was related to the suppression of AKT and p38 expressions.
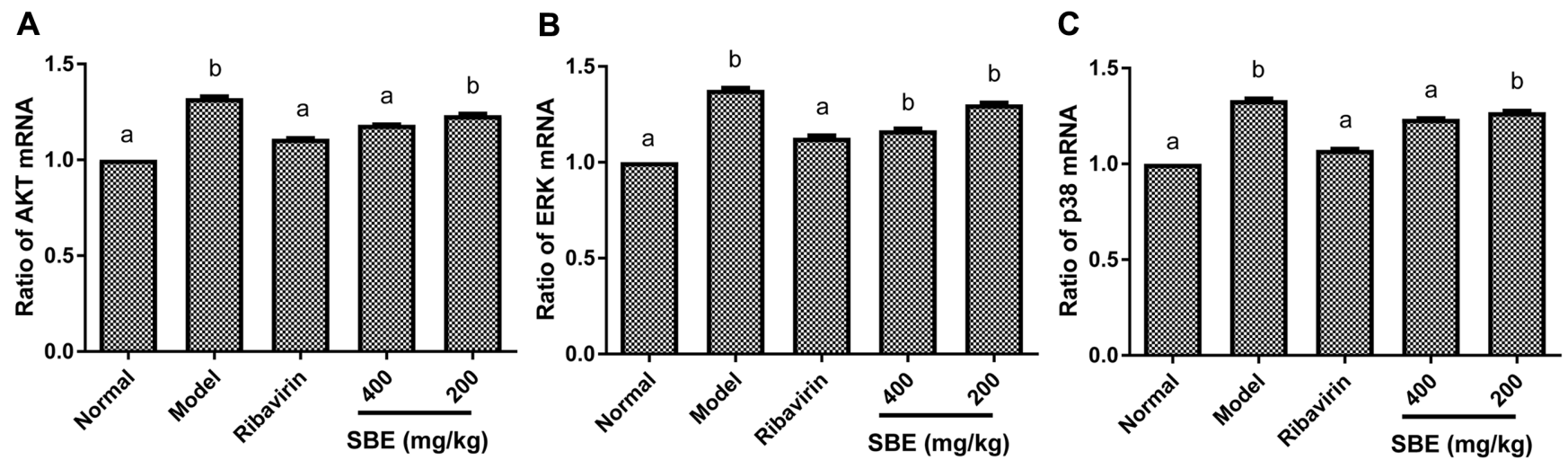

Fig. 6. Effects of SBE on mRNA levels of AKT, ERK, and p38 in CVB3 myocarditis mice model.

The data shown are the RT-PCR results of relative mRNA levels of (A) AKT, (B) ERK, and (C) p38 to Normal group treated with ribavirin $(100 \mathrm{mg} / \mathrm{kg})$ and SBE (400 and $200 \mathrm{mg} / \mathrm{kg})$ for $28 \mathrm{~d}$, respectively. Different letters represent significant differences between two groups $(p<0.05)$. 

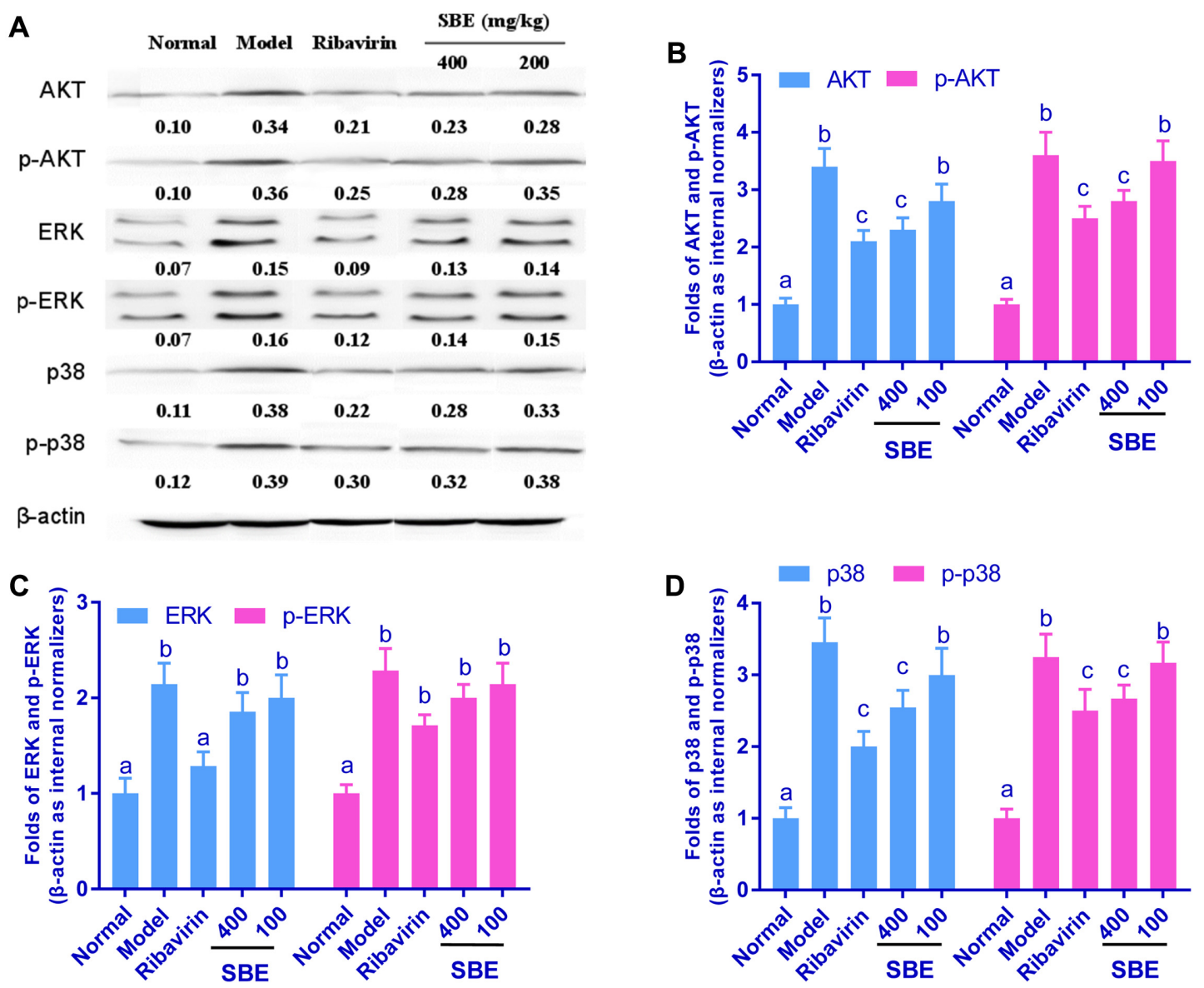

Fig. 7. SBE inhibited CVB3-induced activation of AKT, ERK and p38 in myocarditis mice model.

(A) Representative picture of total and phosphorylated AKT, ERK and p38 protein expression. Quantitative data of AKT (B), ERK (C), and p38 (D) protein expressions. Data are expressed as fold over control ( $n=3$ per condition). Myocarditis mice were treated with ribavirin $(100 \mathrm{mg} / \mathrm{kg})$ and SBE (400 and $200 \mathrm{mg} / \mathrm{kg}$ ) for $28 \mathrm{~d}$. The levels of AKT, p-AKT, ERK, p-ERK, p38, and p-p38 proteins in heart tissues were analyzed by western blot. $\beta$-actin was used as a loading control. Different letters represent significant differences between two groups $(p<0.05)$.

\section{Discussion}

Scutellaria baicalensis has been used for treating colds, cancer, inflammation, infectious disease, and respiratory infections in China [8]. It was reported that Scutellaria baicalensis extracts (SBE) exhibited anti-dengue virus, antiH1N1 virus, and anti-EV71 virus activity [13-15]. It is an important part of multi-herb formulations such as Xiaochai Hutang, San Wu Huangqin Decoction, and Shuang-HuangLian Injectable Powder, which can be used as antiviral agents against influenza virus and human adenovirus III $[16,17]$. The present study investigated the therapeutic effect of Scutellaria baicalensis extracts (SBE) on CVB3induced myocarditis. We first found that SBE possessed anti-CVB3 activity both in vitro and in vivo.

Viral myocarditis is a common illness in infants, children and young adults and can induce cardiomyocyte necrosis and degeneration. CVB3 is the most common viral cause of heart failure, which results in irreversible cytopathic effects at the cellular level and cardiac injury at the tissue level. Pathogenic mechanisms include direct CVB3-induced damage to the heart tissue, host-cell inflammatory responses to the viral infection or a mixture of these two, which may synergistically promote cardiac toxicity [18]. Several signaling pathways may be involved in the process of CVB3 infection along with activation of caspase-3, activation of $\mathrm{ERK}_{1 / 2}$, activation of $\mathrm{PKB} / \mathrm{AKT}$, ER stress, p38 MAPK and PI3K/AKT/mTOR [5-7]. To identify the mechanisms of the effect of SBE on CVB3-induced viral myocarditis, we determined the expression of AKT, ERK, and p38 genes and proteins by RT-PCR and western blot methods. 
Some reports showed that the upregulation of AKT, ERK, and p38 signaling pathways in CVB3-infected host cells such as HeLa cells and rat cardiac fibroblasts [6, 19, 20]. AKT is associated with apoptosis and autophagy processes in CVB3-infected cells [5, 19]. Both ERK and p38 are types of MAPKs, which play a major role in myocarditis. CVB3 can up-regulate the ERK pathway in myocardial microvascular endothelial cells [6] but not in HeLa cells [20]. p38 is the downstream kinase of AMPK in CVB3infected neonatal rat cardiac fibroblasts, which influences the inflammatory cardiomyopathy and myocardial fibrosis induced by CVB3 [7]. The activation of p38 is also found in HeLa cells and primary myocardial cells after CVB3 infection [20]. In the present study, rat primary myocardial cells were selected as the host cells of CVB3. Consistent with the reported results, the levels of AKT, ERK, and p38 mRNA in primary myocardial cells were enhanced after CVB3 infection, as well as the p-AKT, p-ERK and p-p38 proteins. SBE ameliorated CVB3-induced cytotoxicity in myocardial cells by suppression of AKT and p38 activation without influencing normal cell proliferation (Figs. 1-3). In the animal model of CVB3 viral myocarditis, the model was confirmed by the rate of mortality and pathologic changes in the myocardial tissue. Heart tissues were significantly increased in CVB3 viral myocarditis model group. SBE treatment resulted in a marked reduction in the mortality and severity of myocarditis (Fig. 4). We found that $\mathrm{SBE}$ can reduce the $\mathrm{AKT}$ and $\mathrm{p} 38$ genes expression in cardiac muscle tissue (Fig. 5). Consistently, the expression of AKT and p38 proteins were down-regulated when treated with SBE compared to the viral myocarditis model (Fig. 6). Although the therapeutic effect of Scutellaria baicalensis or SBE on CVB3-induced myocarditis has not been studied, it is reported that Scutellaria baicalensis or SBE can inhibit inflammation by PI3K/AKT or ERK pathway in other diseases $[9,10]$. Our results indicate that anti-CVB3 viral myocarditis activity of SBE is likely achieved by modulating AKT and $\mathrm{p} 38$ pathways.

SBE as recorded in Chinese pharmacopeia is the aqueous extracts of Scutellaria baicalensis, which mostly contains baicalin (85\%). Baicalin as a bioactive compound showed various activities including anti-virus, anti-cancer, antiinflammatory, anti-atherosclerosis and so on [21-26]. The effects of baicalin on the disease are relative with the activating AMPK, p38, AKT, and ERK signaling pathways. However, the anti-CVB3 activity of baicalin has not been reported. In addition to baicalin, baicalein, wogonin, and oroxylin A are also main elements of Scutellaria baicalensis, which also exhibit similar activities. With respect to the anti-CVB3 activity, only oroxylin A was reported to protect Vero cells against CVB3-induced cell death. As for antiviral activity, wogonin has anti-influenza viral activity by AMPK pathway, and baicalein inhibits the growth of Epstein-Barr virus positive nasopharyngeal carcinoma [11, $27,28]$. Therefore, we hypothesized that all the compounds of baicalin, baicalein, wogonin, and oroxylin A of SBE can interact with each other and play a role in anti-CVB3 virus activity from different signaling pathways, which needs further investigation. To the best of our knowledge, this is the first demonstration that SBE has an inhibitory effect on CVB3 viral-induced myocarditis, and it may downregulate the expression of AKT and p38 in viral infected primary myocardial cells and viral myocarditis animal model.

In the current study, we have demonstrated that SBE has anti-CVB3 properties both in vitro and in vivo. Additionally, SBE can repair tissue injury and prolong survival in mice with viral myocarditis. However, the exact compounds and the molecular mechanisms by which SBE mediates these antiviral effects against CVB3 remain to be elucidated. Therefore, our findings indicate that SBE is a promising potent antiviral agent with a substantial impact on the survival and pathological changes in CVB3-induced myocarditis.

\section{Acknowledgment}

This work was supported by the National Science Foundation of China (31501056), and the Natural Science Foundation of Tianjin (16JCQNJC09000).

\section{Conflict of Interest}

The authors have no financial conflicts of interest to declare.

\section{Reference}

1. Sagar S, Liu PP, Cooper LT, Jr. 2012. Myocarditis. Lancet 379: 738-747.

2. Pollack A, Kontorovich AR, Fuster V, Dec GW. 2015. Viral myocarditis-diagnosis, treatment options, and current controversies. Nat. Rev. Cardiol. 12: 670-680.

3. Howlett SE. 2018. Coxsackievirus B3-induced myocarditis: new insights into a female advantage. Can. J. Cardiol. 34: 354-355.

4. Rose NR. 2014. Learning from myocarditis: mimicry, chaos and black holes. F1000Prime Rep. 6: 25. 
5. Chang H, Li X, Cai Q, Li C, Tian L, Chen J, et al. 2017. The $\mathrm{PI} 3 \mathrm{~K} / \mathrm{Akt} / \mathrm{mTOR}$ pathway is involved in CVB3-induced autophagy of HeLa cells. Int. J. Mol. Med. 40: 182-192.

6. Wen J, Huang C. 2017. Coxsackieviruses B3 infection of myocardial microvascular endothelial cells activates fractalkine via the ERK1/2 signaling pathway. Mol. Med. Rep. 16: 75487552.

7. Jiang S, Jiang D, Zhao P, He X, Tian S, Wu X, et al. 2016. Activation of AMP-activated protein kinase reduces collagen production via p38 MAPK in cardiac fibroblasts induced by coxsackievirus B3. Mol. Med. Rep. 14: 989-994.

8. Zhao Q, Chen XY, Martin C. 2016. Scutellaria baicalensis, the golden herb from the garden of Chinese medicinal plants. Sci. Bull. 61: 1391-1398.

9. Li Q, Li Y, Li J, Ma Y, Dai W, Mo S, et al. 2018. FBW7 suppresses metastasis of colorectal cancer by inhibiting HIF1alpha/CEACAM5 functional axis. Int. J. Biol. Sci. 14: 726-735.

10. Hussain I, Waheed S, Ahmad KA, Pirog JE, Syed V. 2018. Scutellaria baicalensis targets the hypoxia-inducible factor1alpha and enhances cisplatin efficacy in ovarian cancer. $J$. Cell Biochem. 119: 7515-7524.

11. Kwon BE, Song JH, Song HH, Kang JW, Hwang SN, Rhee $\mathrm{KJ}$, et al. 2016. Antiviral activity of oroxylin A against Coxsackievirus B3 alleviates virus-induced acute pancreatic damage in mice. PLoS One 11: e0155784.

12. Jiang Y, Zhu Y, Mu Q, Luo H, Zhi Y, Shen X. 2017. Oxymatrine provides protection against Coxsackievirus B3induced myocarditis in BALB/c mice. Antiviral Res. 141: 133-139.

13. Zandi K, Lim TH, Rahim NA, Shu MH, Teoh BT, Sam SS, et al. 2013. Extract of Scutellaria baicalensis inhibits dengue virus replication. BMC Complement. Altern. Med. 13: 91.

14. Ji S, Li R, Wang Q, Miao WJ, Li ZW, Si LL, et al. 2015. AntiH1N1 virus, cytotoxic and Nrf2 activation activities of chemical constituents from Scutellaria baicalensis. J. Ethnopharmacol. 176: $475-484$.

15. Lin $\mathrm{H}$, Zhou J, Lin $\mathrm{K}$, Wang $\mathrm{H}$, Liang Z, Ren X, et al. 2016. Efficacy of Scutellaria baicalensis for the treatment of hand, foot, and mouth disease associated with Encephalitis in patients infected with EV71: a multicenter, retrospective analysis. Biomed. Res. Int. 2016: 5697571.

16. Ma Q, Liang D, Song S, Yu Q, Shi C, Xing X, et al. 2017. Comparative study on the antivirus activity of ShuangHuang-Lian injectable powder and its bioactive compound mixture against human adenovirus III in vitro. Viruses 9(4): 79.
17. Ma Q, Yu Q, Xing X, Liu S, Shi C, Luo J. 2018. San Wu Huangqin Decoction, a Chinese herbal formula, inhibits influenza a/PR/8/34 (H1N1) virus infection in vitro and in vivo. Viruses 10(3): 117.

18. Garmaroudi FS, Marchant D, Hendry R, Luo H, Yang D, Ye X, et al. 2015. Coxsackievirus B3 replication and pathogenesis. Future Microbiol. 10: 629-653.

19. Lee YG, Park JH, Jeon ES, Kim JH, Lim BK. 2016. Fructus amomi cardamomi extract inhibit coxsackievirus-B3 induced myocarditis in murine myocarditis model. J. Microbiol. Biotechnol. 26: 2012-2018.

20. Dai Q, Zhang D, Yu H, Xie W, Xin R, Wang L, et al. 2017. Berberine restricts coxsackievirus $\mathrm{B}$ type 3 replication via inhibition of c-Jun N-terminal kinase (JNK) and p38 MAPK activation in vitro. Med. Sci. Monit. 23: 1448-1455.

21. Chen Y, Yuan W, Yang Y, Yao F, Ming K, Liu J. 2018. Inhibition mechanisms of baicalin and its phospholipid complex against DHAV-1 replication. Poult. Sci. 97: 3816-3825.

22. Chen Y, Yang Y, Wang F, Yang X, Yao F, Ming K, et al. 2018. Antiviral effect of baicalin phospholipid complex against duck hepatitis A virus type 1. Poult. Sci. 97: 2722-2732.

23. Kang S, Liu S, Li H, Wang D, Qi X. 2018. Baicalin effects on rats with spinal cord injury by anti-inflammatory and regulating the serum metabolic disorder. J. Cell Biochem. 119: 7767-7779.

24. Acta virologicaWu Y, Wang F, Fan L, Zhang W, Wang T, $\mathrm{Du} \mathrm{Y}$, et al. 2018. Baicalin alleviates atherosclerosis by relieving oxidative stress and inflammatory responses via inactivating the NF-kappaB and p38 MAPK signaling pathways. Biomed. Pharmacother. 97: 1673-1679.

25. You J, Cheng J, Yu B, Duan C, Peng J. 2018. Baicalin, a Chinese herbal medicine, inhibits the proliferation and migration of human non-small cell lung carcinoma (NSCLC) cells, A549 and H1299, by activating the SIRT1/AMPK signaling pathway. Med. Sci. Monit. 24: 2126-2133.

26. Wang Z, Ma L, Su M, Zhou Y, Mao K, Li C, et al. 2018. Baicalin induces cellular senescence in human colon cancer cells via upregulation of DEPP and the activation of Ras/ Raf/MEK/ERK signaling. Cell Death Dis. 9: 217.

27. Seong RK, Kim JA, Shin OS. 2018. Wogonin, a flavonoid isolated from Scutellaria baicalensis, has anti-viral activities against influenza infection via modulation of AMPK pathways. Acta Virol. 62: 78-85.

28. Zhang Y, Wang H, Liu Y, Wang C, Wang J, Long C, et al. 2018. Baicalein inhibits growth of Epstein-Barr virus-positive nasopharyngeal carcinoma by repressing the activity of EBNA1 Q-promoter. Biomed. Pharmacother. 102: 1003-1014. 